\title{
Non-monotonous growth and motion of the South Atlantic Anomaly
}

Hagay Amit ( $\nabla$ hagay.amit@univ-nantes.fr )

Universite de Nantes

\section{Filipe Terra-Nova}

Universidade de Sao Paulo

Maxime Lézin

Universite de Nantes

Ricardo Trindade

Universidade de Sao Paulo

Full paper

Keywords: Geomagnetic field, secular variation, South Atlantic Anomaly, core-mantle boundary

Posted Date: April 28th, 2020

DOl: https://doi.org/10.21203/rs.3.rs-24657/v1

License: (c) (i) This work is licensed under a Creative Commons Attribution 4.0 International License. Read Full License 


\section{Non-monotonous growth and motion of the South Atlantic Anomaly}

${ }_{3} \quad$ Hagay Amit ${ }^{a, *}$, Filipe Terra-Nova ${ }^{b}$, Maxime Lézin ${ }^{a}$, Ricardo I. Trindade $^{b}$

$5{ }^{a}$ CNRS UMR 6112, Université de Nantes, Laboratoire de Planétologie et de Géodynamique,

62 rue de la Houssinière, Nantes, F-44000, France

$7{ }^{b}$ Departamento de Geofísica, Instituto de Astronomia, Geofísica e Ciências Atmosféricas,

8 Universidade de São Paulo, Rua do Matão, 1226, Cidade Universitária, 05508-090 São Paulo,

9 Brazil

${ }_{10}^{*}$ Corresponding author

11 Email addresses: Hagay.Amit@univ-nantes.fr (H. A.), filipeterranova@iag.usp.br (F. T-N.),

12 maxime.lezin@etu.univ-nantes.fr(M.L.), ricardo.trindade@iag.usp.br (R.I.T.) 
and rapid southward drift. We interpret these special events in terms of the secular variation of relevant large-scale geomagnetic flux patches on the core-mantle boundary. Our characterization may be used as a constraint on Earth-like numerical dynamo models.

Keywords: Geomagnetic field, secular variation, South Atlantic Anomaly, core-mantle boundary

\section{Introduction}

The South Atlantic Anomaly (SAA) is a region at Earth's surface where the intensity of the magnetic field is particularly low. This leads to penetration of solar energetic particles deep into Earth's atmosphere, posing severe problems for airplanes and ships positioning systems as well as spacecraft electronic systems (e.g. Olson and Amit, 2006). Understanding the past and present locations and mobility as well as the future trajectory of the SAA is both a fundamental scientific challenge - it involves understanding the working of the geodynamo and the impact of core-mantle coupling on core dynamics, as well as an important societal issue - it has major consequences for the operation and protection of surface instruments and spacecrafts, from global positioning systems to the Hubble Space Telescope, which cannot obtain observations over the SAA region.

The current location of the SAA center in Brazil is related to the location of reversed geomagnetic flux patches (RFPs) at the core-mantle boundary (CMB) (Olson and Amit, 2006) though this relation is not trivial (Terra-Nova et al., 2017). It is under debate whether the current SAA location represents a long-term feature of Earth's magnetic field or it is chaotically variable. Based on a data assimilation scheme, Aubert (2015) predicted that the SAA will drift in the near future to the Pacific, i.e. it is a transient feature of the geodynamo. In contrast, some archeomagnetic field models exhibit persistent minimum surface intensity in the South Atlantic (Brown et al., 2018; Hellio and Gillet, 2018). Campuzano et al. (2019) found that the SAA has been expanding and westward drifting since 1400. In addition, based on archeological materials, it was argued that the SAA has influenced the surface geomagnetic field for several millennia in Africa (Tarduno et al., 2015; Hare et al., 2018) and South America (Trindade et al., 2018; Hartmann et al., 2019). Tarduno et al. (2015) used such local intensity timeseries 
to propose that topographic core-mantle coupling has fixated the SAA. However, inferring spatial minima from local intensity timeseries might be erroneous. Overall, although new archeomagnetic data for the South Atlantic continue to be acquired, the record is still far from being satisfactory.

Heterogeneous CMB conditions may affect the morphologies of outer core convection and the induced geomagnetic field. Numerical dynamo simulations with imposed tomographic outer boundary heat flux have been widely applied to explore geodynamo features, most commonly preferred locations of intense geomagnetic flux patches on the CMB (Gubbins et al., 2007; Aubert et al., 2008; Amit et al., 2015). Terra-Nova et al. (2019) applied such models to show that the longitude of the SAA center may be mantle controlled; However, recovering its relatively large latitude remains a challenge.

Geomagnetic field models spanning the historical era (e.g. Jackson et al., 2000) and more recent modern periods (e.g. Finlay et al., 2010) may provide reliably the location and mobility of the SAA. However, the SAA characterization has been somewhat crude. In this paper we will show that SAA area estimates based on a fixed thershold (De Santis et al., 2013; PavónCarrasco and De Santis, 2016; Campuzano et al., 2019) are strongly affected by global axial dipole variations and as such do not represent adequately regional morphological changes. Furthermore, SAA center estimates based on surface minimum positions (e.g. Terra-Nova et al., 2017) do not take into account anisotropic SAA shape. In order to better understand the kinematic origin of the SAA and constrain numerical dynamos (Terra-Nova et al., 2019), a precise and reliable characterization of the SAA in terms of its area and center is required.

Previous studies characterized the SAA at altitudes of $\sim 800 \mathrm{~km}$ above Earth's surface, corresponding to low-Earth orbits of satellites (Casadio and Arino, 2011; Schaefer et al., 2016; Anderson et al., 2018). Here we characterize the SAA at Earth's surface where geomagnetic observations have been continuously acquired since the advent of intensity measurement (Jackson et al., 2000). We then explore the outer core kinematic origin of the SAA.

The paper is outlined as follows. In section 2 we introduce and justify our new measures of the SAA area and center. The results for this SAA characterization are presented in section 3 , and the kinematic interpretations in terms of temporal evolution of relevant flux patches on 


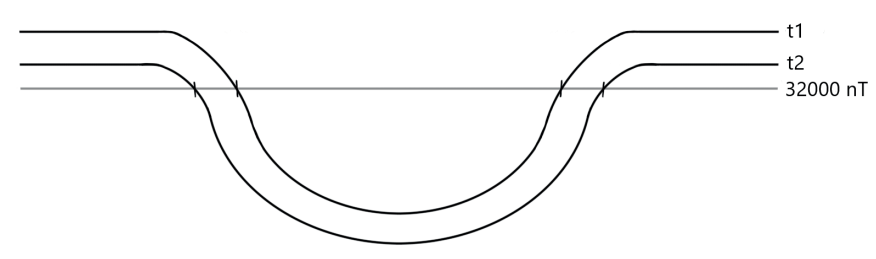

Figure 1: Schematic illustration of a cross-section of the surface field intensity under a global decrease in field intensity from time $t 1$ to time $t 2$. In this scenario, the apparent SAA area based on $S 0$ (1) increases.

the CMB are given in section 4 . We conclude our main findings in section 5.

\section{${ }_{83} 2$ Method}

We compare the characterization of the SAA based on previous studies vs. our proposed measures. This characterization includes both the area and the coordinates of the SAA center.

Previous studies defined the SAA area as that where the geomagnetic field intensity $|\vec{B}|$ at Earth's surface is lower than 32000 nT (De Santis et al., 2013; Pavón-Carrasco and De Santis, 2016):

$$
|\vec{B}|<32000 \mathrm{nT}
$$

From hereafter we term the area based on (1) as $S 0$. This definition is practical for space safety purposes. However, from a more fundamental point of view, (1) is affected not only by regional spatio-temporal field variations, but also by global changes, such as the historical geomagneic dipole decrease (e.g. Gubbins, 1987; Olson and Amit, 2006; Finlay, 2008; Huguet et al., 2018). Fig. 1 illustrates this point. Under a hypothetical scenario of entire field magnitude decrease with no pattern change, a fixed critical threshold (such as $32000 \mathrm{nT}$ ) for the SAA would suggest that its area increases despite no regional variation.

To overcome this problem, alternatively we factor the critical intensity value by the instantaneous axial dipole $g_{1}^{0}(t)$ normalized by its value at the middle of the investigated period:

$$
|\vec{B}|<32000 \frac{g_{1}^{0}(t)}{g_{1}^{0}(1930)} \mathrm{nT}
$$


From hereafter we term the area based on (2) as $S 1$. With this definition the critical value varies with the axial dipole. As such, it captures the regional variation of the SAA area, independently of the change in the global field magnitude.

Next, previous studies tracked the SAA center based on the point of minimum field intensity, both at Earth's surface (Hartmann and Pacca, 2009; Finlay et al., 2010; Aubert, 2015; Terra-Nova et al., 2017, 2019) as well as at higher altitudes (Anderson et al., 2018). Following Terra-Nova et al. (2017), we reproduce this result by first searching for the grid point with lowest intensity and then applying second order polynomial interpolations using two neighboring points in each direction to resolve off-grid values. From hereafter we term these coordinates as Min. This minimum intensity genuinely represents the center of the SAA only if its area is isotropic. However, if the shape is significantly anisotropic, the minimum point might not well represent the center of the structure (for an illustration see Fig. 2).

Alternatively, centers of mass were invoked to identify and track centers of intense flux patches on the CMB in numerical dynamos (Amit et al., 2010) and geomagnetic field models (Amit et al., 2011). Centers of mass were also used to identify the SAA at $\sim 800 \mathrm{~km}$ altitude (Casadio and Arino, 2011; Schaefer et al., 2016). Here we calculate centers of mass to determine the longitude and co-latitude of the SAA center, $\phi_{c m}$ and $\theta_{c m}$ respectively, at Earth's surface:

$$
\phi_{c m}=\frac{\sum_{S} \phi_{i} w_{i}}{\sum_{S} w_{i}}
$$

$$
\phi_{c m}=\frac{\sum_{S} \theta_{i} w_{i}}{\sum_{S} w_{i}}
$$

The summations in (3)-(4) are over the SAA area, either $S 0$ or $S 1$, which we term $C M 0$ and $C M 1$ respectively. The weight $w$ is given by the inverse of the intensity

$$
w_{i}=\frac{1}{\left|\vec{B}_{i}\right|}
$$

Determining the center of the SAA based on the center of mass of its area well represents the center even if its shape is significantly anisotropic.

Both areas $S 0$ and $S 1$ were calculated using a simple trapezoid numerical scheme. Tests of the dependence of the results on the grid size show very weak sensitivity and fast convergence 


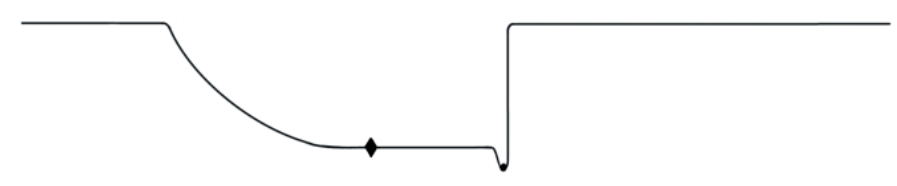

Figure 2: Schematic illustration of a cross-section of the surface field intensity with an anisotropic configuration. The SAA center based on the minimum (Min, denoted by a point) does not represent well the center of the anomaly. The center of mass method CM2 gives an adequate center denoted by a diamond.

with increasing resolution. For all calculations we used a $1^{\circ} \times 1^{\circ}$ grid in longitude and colatitude. With this grid size the computed properties (i.e. the area and coordinates of the center) practically reach asymptotic values with decreasing grid size.

\section{Secular variation of the area and center of the South At- lantic Anomaly}

We used the COV-OBS.x1 time-dependent geomagnetic field model (Gillet et al., 2015) for the period 1840-2020. This model is advantageous for two main reasons. First, it covers the entire historical period, allowing to avoid different field models constructed based on different methods for different epochs (as was previously done in the SAA context by e.g. Pavón-Carrasco and De Santis, 2016; Terra-Nova et al., 2017). Second, COV-OBS.x1 is an ensemble of 100 models, allowing to assess the uncertainty of the results due to the uncertainty of the geomagnetic observations (again in contrast to Pavón-Carrasco and De Santis, 2016; Terra-Nova et al., 2017).

Fig. 3 shows the intensity of the geomagnetic field at Earth's surface for four snapshots. Before 1930 (Fig. 3 top) the SAA area based on $S 0$ (green dashed contour) was smaller than the area based on $S 1$ (purple dashed contour), whereas after 1930 (Fig. 3 bottom) the area based on $S 0$ was larger. This is trivially expected from the definition of $S 1$ (2). However, the visible differences between $S 0$ and $S 1$ in Fig. 3 are remarkable, providing testimony to the large contribution of the axial dipole decrease to the apparent increase of the SAA area. The 

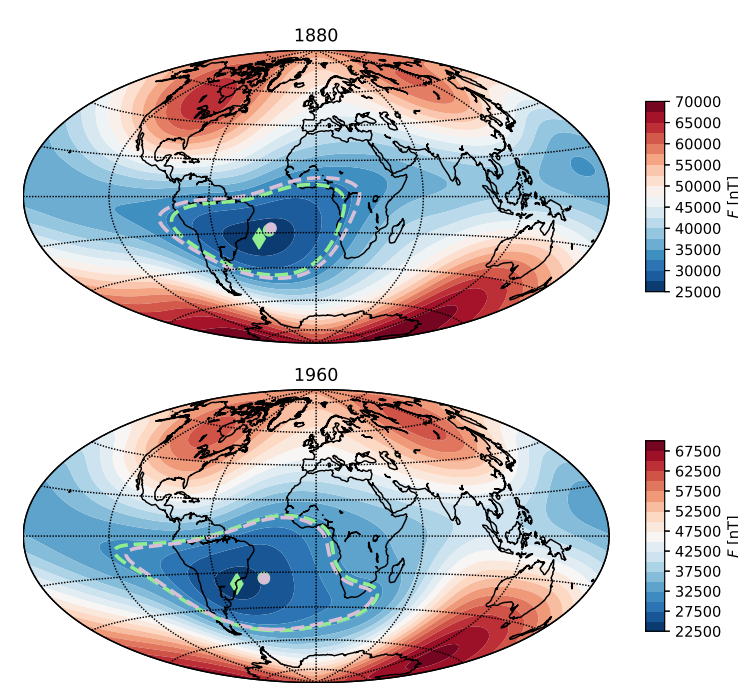

centers of the SAA based on $M i n, C M 0$ and $C M 1$ are very similar at early snapshots when the SAA area was rather isotropic. However, towards present day the shape of the SAA became more complex with thin branches extending to equatorial east Pacific and South Africa. This anisotropic shape produced significantly different centers for $\mathrm{Min}, C M 0$ and $C M 1$.
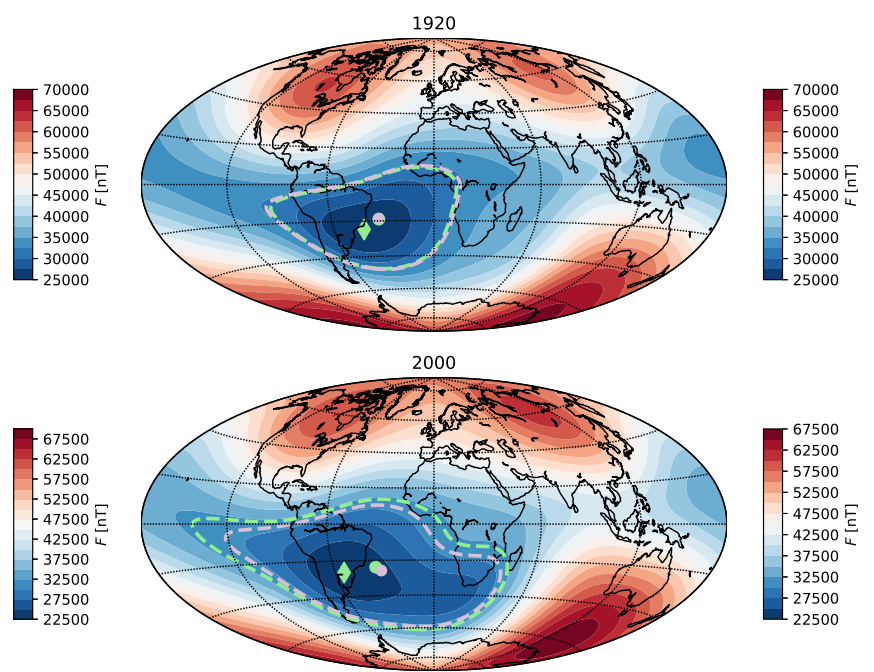

Figure 3: Geomagnetic field intensity at Earth's surface for the mean model of COV-OBS.x1 at four snapshots (Gillet et al., 2015). Dashed green contours denote the $S 0$ area, dashed purple contours denote the $S 1$ area. Green diamonds denote the Min center, green and purple diamonds denote the centers of mass $C M 0$ and $C M 1$, respectively. Note the different scales from top to bottom.

Fig. 4 quantifies the characterization of the SAA over the entire period 1840-2020. Overall the minimum intensity (Fig. 4a) has been monotonically decreasing, except for the period 1880-1920 when it deviated from the overall linear trend. The increase of the SAA area (Fig. 4b) based on $S 0$ (yellow) is also quite monotonic. Less so is the evolution of $S 1$ (green), including a period between $\sim 1890-1940$ (see dashed vertical lines in Fig. 4) when $S 1$ was flat and even decreasing. The longitude of the SAA center (Fig. 4c) based on Min (purple) has been monotonically decreasing, corresponding to a westward drift. In contrast, the SAA center longitudes based on $C M 0$ (yellow) and $C M 1$ (green) are more variable. Most of the time the SAA based on these two models have also been drifting westward, but significantly slower. Moreover, between $\sim 1940-1980 C M 0$ and $C M 1$ have been drifting eastward. The latitude of the SAA center (Fig. 4d) has been decreasing, corresponding to a southward drift. According 
(a)

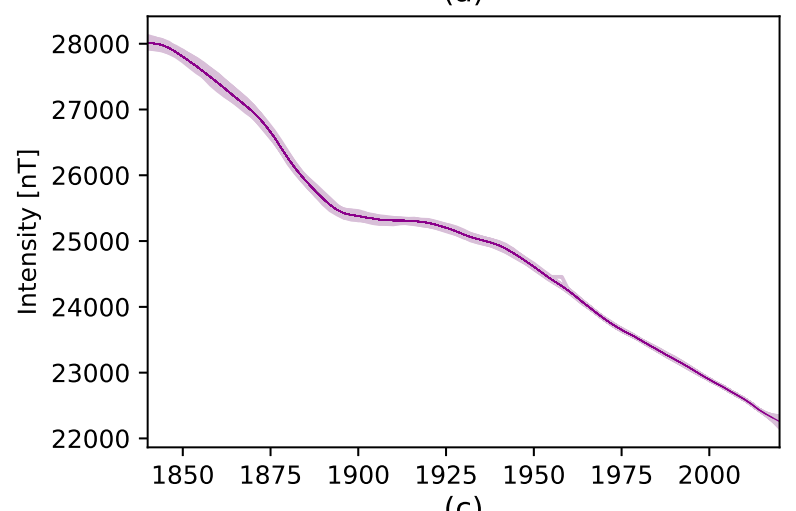

(c)

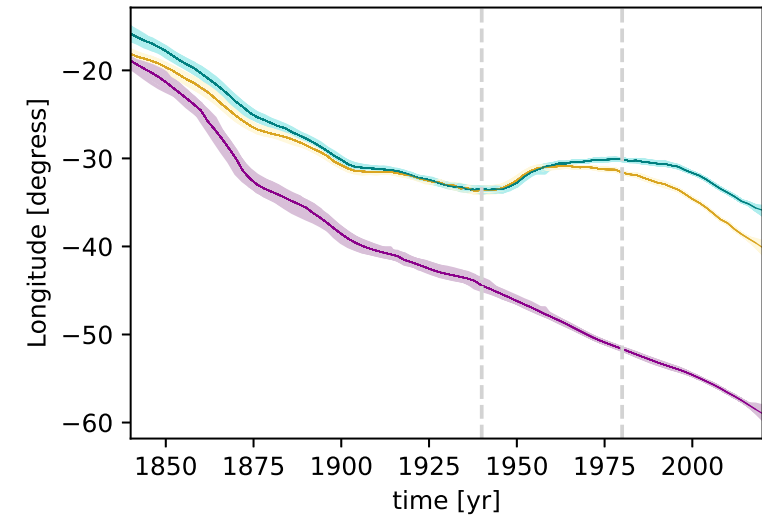

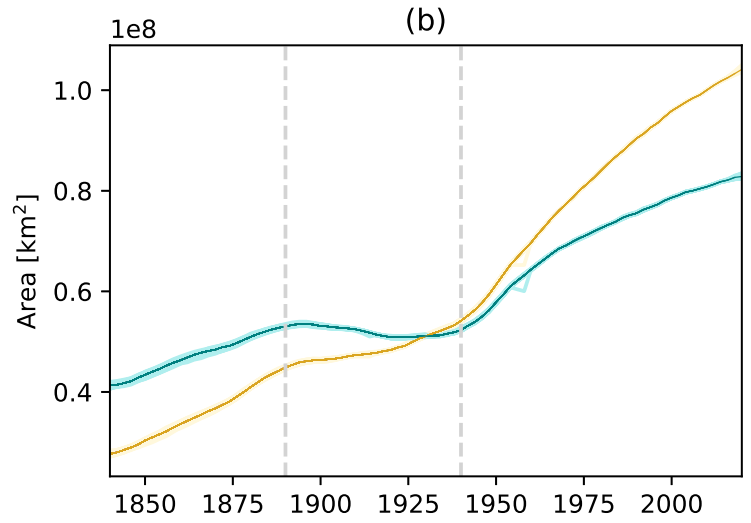

(d)

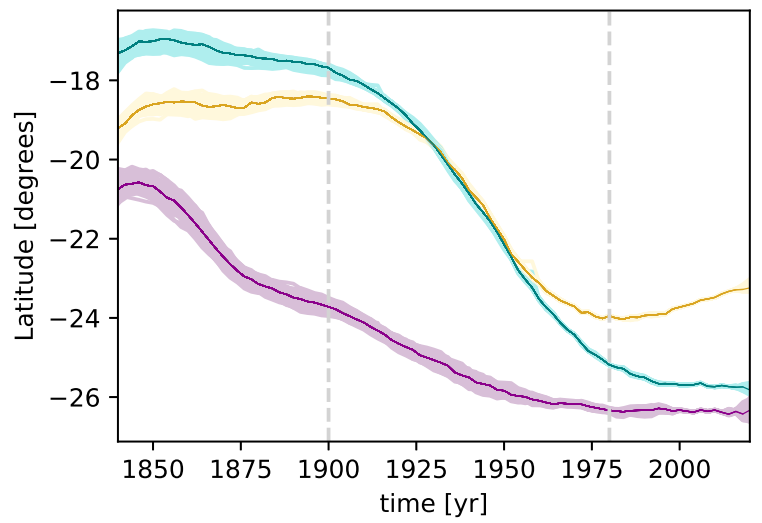

Figure 4: SAA characterization vs. time for the period 1840-2020. Dark lines denote the mean model of COV-OBS.x1, light envelopes denote the 100 realizations of the ensemble. (a) Minimum intensity; (b) Area based on $S 0$ (yellow) and $S 1$ (green); (c) Longitude of center based on Min (purple), $C M 0$ (yellow) and $C M 1$ (green); (d) Latitude of center based on Min (purple), $C M 0$ (yellow) and $C M 1$ (green). Vertical dashed lines highlight special events of SAA area decrease (b), SAA center eastward drift (c) and SAA center rapid southward drift (d).

Fig. 4 also shows the results for the ensemble of all 100 realizations of COV-OBS.x1 (light 
albeit slightly thicker at early periods. Clearly the SAA, being a surface property, is weakly sensitive to the uncertain small-scale field. We therefore conclude that the results in Fig. 4 are robust and insensitive to uncertainties in the field model.

Table 1 presents the rates of change of the SAA area and coordinates of center based on the various models. More specifically, in Table 1 we compare the rates of changes for the periods when the above mentioned special events were captured by our $S 1$ and $C M 1$ preferred models vs. the more 'typical' periods, i.e. when the SAA area increased and its center moved mostly westward with little latitudinal mobility. Our $S 1$ model contains a period of $\sim 50$ years in which the SAA area actually decreased. Moreover, the total rate of change of our $S 1$ model is roughly twice slower than that of the commonly used $S 0$, clearly demonstrating the huge contribution of the global axial dipole decrease to the apparent evolution of the SAA area based on the latter. The total westward drift of model Min is also about twice larger than that of our $C M 1$ model. In addition, according to $C M 1$ the SAA drifted eastward during a period of $\sim 40$ years, whereas $M$ in drifted westward throughout the entire period. Note that unlike the nearly flat SAA area decrease event of $S 1$, the eastward drift event of $C M 1$ is nonnegligible, comparable to its rate of westward drift over the entire period. Finally, according to model Min the SAA drifted southward monotonically and decelerated towards present day. In contrast, our model $C M 1$ shows two periods with little latitudinal change and in between a period of $\sim 80$ years in which the SAA drifted southward in an impressive rate of $0.105 \% \mathrm{yr}$, about three times faster than the average southward drift rate of Min over the entire period. 


\begin{tabular}{ccccc}
\hline Area & $1840-1890$ & $1890-1940$ & $1940-2020$ & Total \\
\hline$S 0$ & 3.51 & 1.71 & 6.38 & 4.19 \\
$S 1$ & 2.43 & -0.46 & 3.76 & 2.22 \\
\hline Center longitude & $1840-1940$ & $1940-1980$ & $1980-2020$ & Total \\
\hline Min & -0.268 & -0.189 & -0.174 & -0.230 \\
$C M 0$ & -0.166 & 0.068 & -0.226 & -0.127 \\
$C M 1$ & -0.189 & 0.105 & -0.157 & -0.117 \\
\hline Center co-latitude & $1840-1900$ & $1900-1980$ & $1980-2020$ & Total \\
\hline Min & -0.059 & -0.034 & 0.000 & -0.035 \\
$C M 0$ & 0.006 & -0.085 & 0.021 & -0.031 \\
$C M 1$ & -0.011 & -0.105 & -0.014 & -0.053 \\
\hline & & & &
\end{tabular}

Table 1: Rates of change of the SAA area in $10^{5} \mathrm{~km}^{2} / \mathrm{yr}$ and of the coordinates of its center in $\% \mathrm{yr}$ for all models. Rates of change are given for specific periods motivated by Fig. 4 (see text) as well as for the entire period (denoted by 'Total').

\section{Outer core kinematic interpretation}

What is the kinematic origin of the special events described above? Fig. 5 shows the radial geomagnetic field and its secular variation (SV) on the CMB. To infer features that are relevant for the large-scale surface field, both quantities are truncated at spherical harmonic degree and order 5 (see e.g. Zossi et al., 2020). To analyze the special events captured by $S 1$ and $C M 1$, we examine three snapshots: 1920 during the SAA area decrease and rapid southward drift; 1960 during the SAA eastward drift and (again) rapid southward drift; and 2000, a 'typical' reference snapshot. Motivated by the localization of the non-linear intensity kernel around the surface observation site (Constable, 2007; Terra-Nova et al., 2017), we centered the maps on the SAA region. For comparison, we also show the corresponding maps truncated at the maximum degree and order 14 of the field model (Fig. 6).

Our below interpretations of the SV of the SAA area and center are guided by the conclusions of Amit (2014): Correlation/anti-correlation between a radial field structure (or flux patch) with an SV structure indicates local intensification/weakening respectively, whereas coincidence of a flux patch with a center of a pair of opposite-sign SV structures suggests local drift. Following Terra-Nova et al. (2017) we focus on the main flux patches of both polarities 

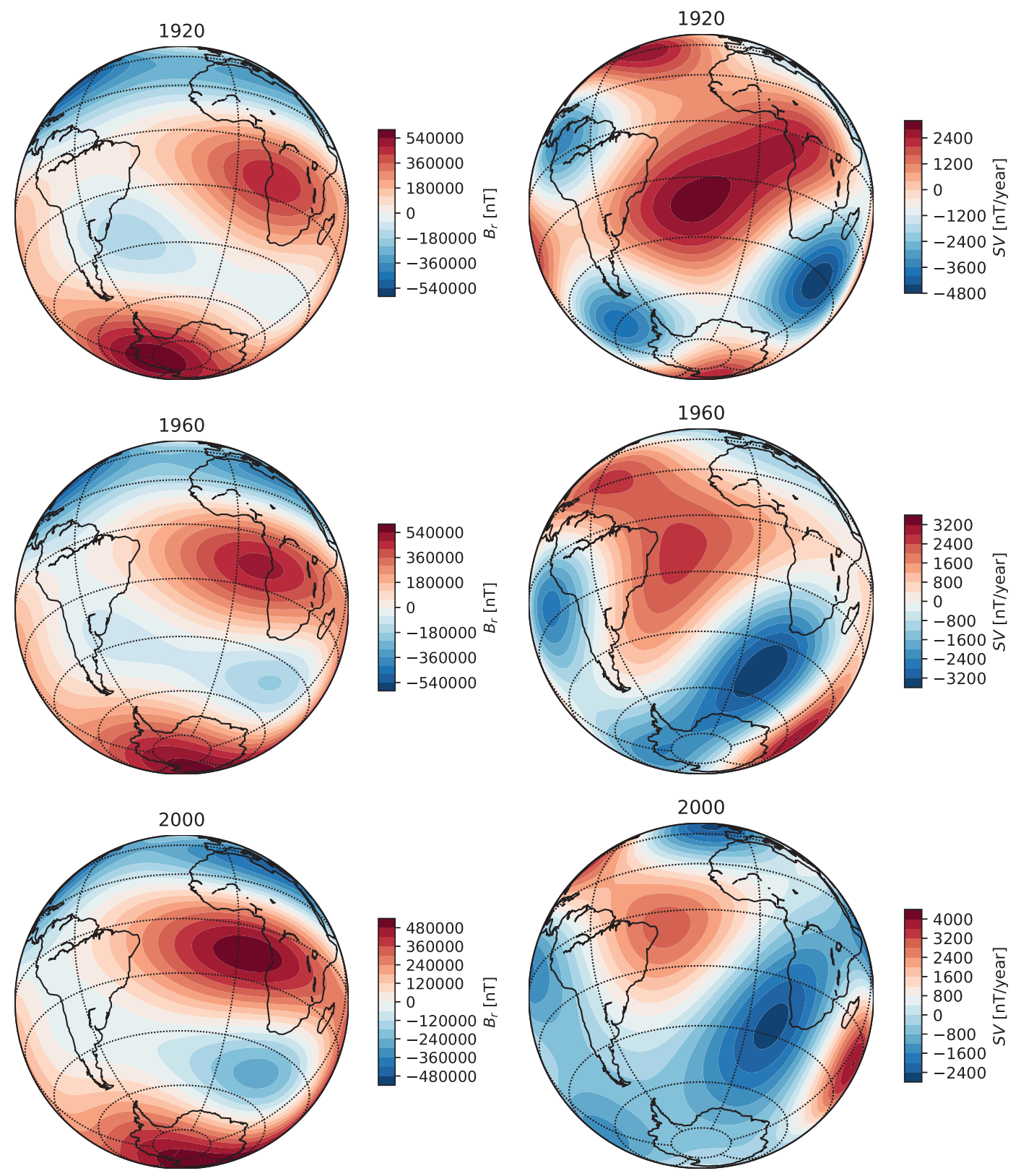

Figure 5: Radial geomagnetic field (left) and its secular variation (right) at the core-mantle boundary for the mean model of COV-OBS.x1 in 1920 (top), 1960 (middle) and 2000 (bottom). All models are expanded until spherical harmonic degree and order 5. All maps are centered at $20^{\circ} \mathrm{W} 30^{\circ} \mathrm{S}$, i.e. on the South Atlantic. Note the different scales.

RFPs and away from NFPs (Terra-Nova et al., 2017, 2019). 

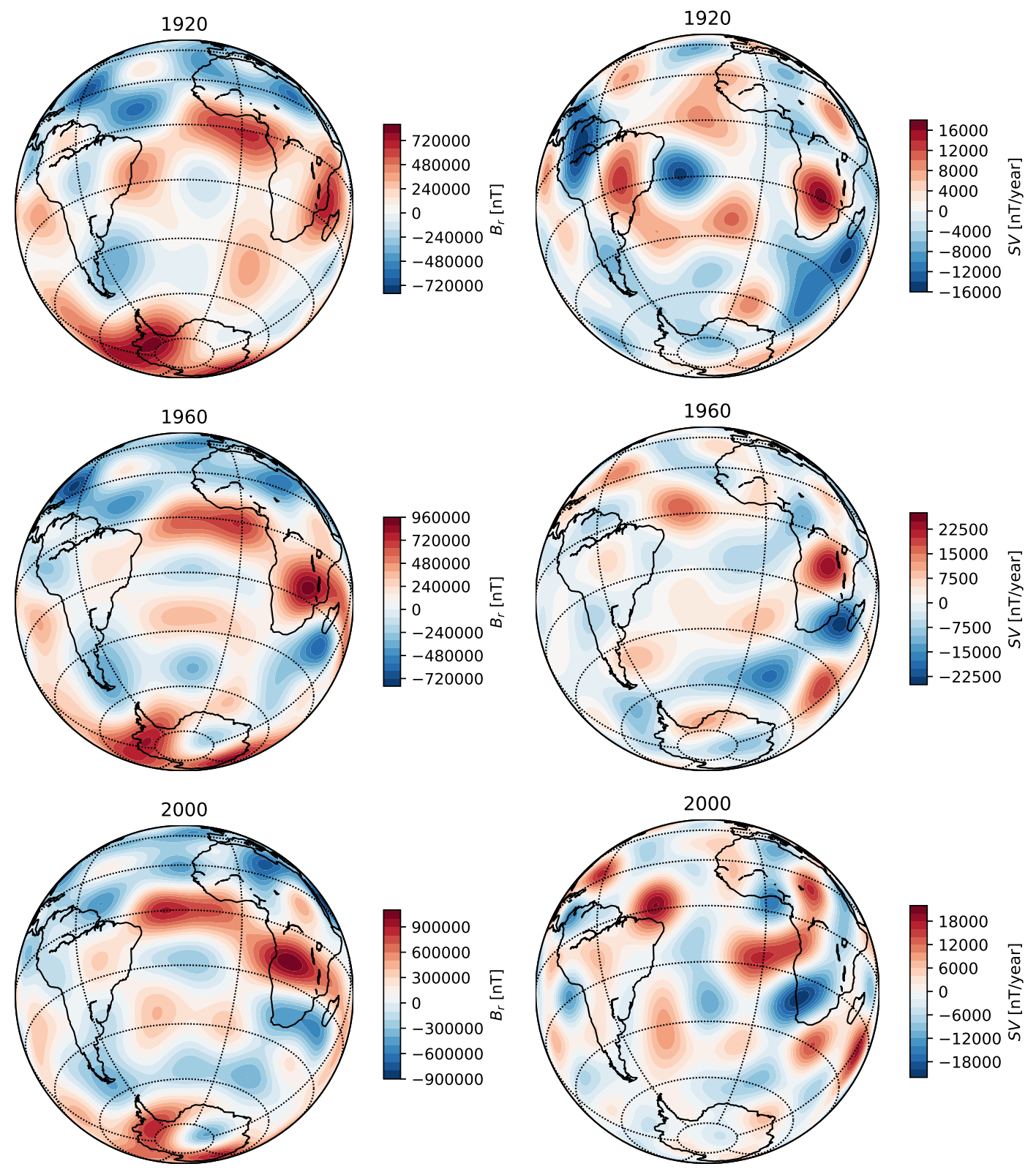

Figure 6: As in Fig. 5 but expanded until spherical harmonic degree and order 14.

The event of SAA area increase (based on $S 1$ ) is related to the SV of the dominating RFPs on the CMB. In 1920, the dominant RFP below Patagonia coincides with a positive (i.e. opposite-sign) SV structure (Fig. 5 top), corresponding to local weakening, hence SAA area increase. In contrast, in 1960 and 2000, the dominant RFP below south of Africa coincides with a same-sign SV structure (Fig. 5 middle and bottom), corresponding to local intensifica- 
tion, hence the 'typical' SAA area decrease.

The SAA eastward drift event (based on $C M 1$ ) can be explained by the SV below midAtlantic between the Patagonia and Africa RFPs. In 1960 a southwest positive SV intrusion extends through mid-Atlantic until the southern tip of Patagonia (Fig. 5 middle), weakening the reversed flux below mid-atlantic. This period marks a transition between the dominant Patagonia RFP in the west Atlantic beforehand (Fig. 5 top) to the dominance of the Africa RFP in the east Atlantic later on (Fig. 5 bottom). The outcome of this transitional period is a brief eastward drift of the SAA.

The SAA rapid southward drift event (again based on $C M 1$ ) is related to the SV of the high-latitude NFP below Antarctica during this period (Fig. 5 top and middle). In 1920 and 1960 this NFP drifted across Antarctica towards the Indian Ocean. Because this NFP is located south of the SAA, its drift away from the South Atlantic led to the rapid southward drift of the SAA. In contrast, in 2000 the SV below this NFP significantly faded (see SV scale in Fig. 5 bottom), resulting in the weak latitudinal motion of the SAA in 2000.

Finding the kinematic origins of the SAA SV in the more detailed maps expanded until spherical harmonic degree and order 14 (Fig. 6) is clearly more challenging. Nevertheless, some of the morphological relations between the radial field and its SV that we identified as the kinematic origins of the special SAA events based on the large-scale maps in Fig. 5 can also be detected in the small-scale counterpart maps in Fig. 6. These include the transition from a dominant RFP below Patagonia in 1920 (Figs. 5 and 6 top) to the emergence of a dominant RFP below Africa (Figs. 5 and 6 bottom). Another example is the dissipation of the NFP below Antarctica (Fig. 6) (see also Terra-Nova et al., 2017).

There are two main differences between our interpretation of the SAA motion to that of Terra-Nova et al. (2017). First, we consider the $C M 1$ model based on the center of mass of the area normalized by the mid-term axial dipole, whereas Terra-Nova et al. (2017) tracked the intensity minimum Min. Second, we analyzed the large-scale field and SV on the CMB which are in general appropriate for any surface application (Zossi et al., 2020), whereas Terra-Nova et al. (2017) explored the full field expanded until degree and order 14. Despite these differences, our results are in decent agreement with those of Terra-Nova et al. (2017), essentially 
pointing to the motions of the major RFPs and the high-latitude NFP below the South Atlantic region as the dominating agents controlling the SAA motion, in particular its deviations from linearity.

\section{Conclusions}

We introduced simple new measures to characterize properly the SAA. Our area calculation accounts for axial dipole changes, thus the resulting SAA area $S 1$ isolates regional morphological variations. Our center calculation is regional (rather than local), thus the resulting SAA center $C M 1$ integrates the effects of SAA anisotropy.

Compared to previous results for the SAA area ( $S 0$; e.g. Pavón-Carrasco and De Santis, 2016) and center (Min; e.g. Terra-Nova et al., 2017), our main findings over the entire historical period are:

- The mean rate of area increase is about twice lower.

- The mean westward drift of the center is also about twice lower.

- However, the mean southward drift is larger.

We identified periods with exceptions to the SAA 'typical' area increase, westward drift and weak latitudinal change. These special events are non-detectable in the previous characterizations, highlighting the strongly time-dependent nature of the SAA. The special events and their kinematic origins are:

- 1890-1940: The SAA area decreased due to weakening of the Patagonia RFP.

- 1940-1980: The SAA center drifted eastward due to transition from early dominance of the RFP below Patagonia at its western limb to later dominance of the RFP below Africa at its eastern limb.

- 1900-1980: The SAA center drifted southward rapidly due to the drift of the highlatitude NFP below Antarctica away from the South Atlantic region. 
It would be interesting to apply our characterization to archeomagnetic field models in order to find whether similar behavior persists over millennial timescales. This characterization may be used to constrain Earth-like numerical dynamos (Christensen et al., 2010; Davies and Constable, 2014; Gastine et al., 2019). Continuous monitoring of Earth's geomagnetic field using surface observatories and dedicated satellite mission (such as the current Swarm constellation) will reveal whether the characterization obtained in this study persists in the future.

\section{Declarations}

\section{Availability of data and materials}

The geomagnetic field model COV-OBS.x1 analysed during the current study is available in the following weblink: http://www.spacecenter.dk/files/magnetic-models/COV-OBSx1/.

\section{Competing interests}

The authors declare that they have no competing interests.

\section{Funding}

H. A. acknowledges financial support from program PNP of INSU. F. T-N. acknowledges Sao Paulo Research Foundation (FAPESP) for grant 2018/07410-3.

\section{Authors' contributions}

F.T-N. and M.L. produced the results. H.A. wrote the paper. All authors read and approved the final manuscript.

\section{Acknowledgments}

H. A. and M. L. thank Guy Moebs and Diana Saturnino for their help. 


\section{References}

Amit, H., 2014. Can downwelling at the top of the earth's core be detected in the geomagnetic secular variation? phys. earth planet. inter. Phys. Earth Planet. Inter. 229, 110-121.

Amit, H., Aubert, J., Hulot, G., 2010. Stationary, oscillating or drifting mantle-driven geomagnetic flux patches? J. Geophys. Res. 115, B07108.

Amit, H., Choblet, G., Olson, P., Monteux, J., Deschamps, F., Langlais, B., Tobie, G., 2015. Towards more realistic core-mantle boundary heat flux patterns: a source of diversity in planetary dynamos. Prog. Earth Planet. Sci. 2:26, DOI: 10.1186/s40645-015-0056-3.

Amit, H., Korte, M., Aubert, J., Constable, C., Hulot, G., 2011. The time-dependence of intense archeomagnetic flux patches. J. Geophys. Res. 116, B12106.

Anderson, P. C., Rich, F. J., Borisov, S., 2018. Mapping the South Atlantic Anomaly continuously over 27 years. J. Atmos. Sol-Terr. Phy. 177, 237-246.

Aubert, J., 2015. Geomagnetic forecasts driven by thermal wind dynamics in the Earth's core. Geophys. J. Int. 203, 1738-1751.

Aubert, J., Amit, H., Hulot, G., Olson, P., 2008. Thermo-chemical wind flows couple Earth's inner core growth to mantle heterogeneity. Nature 454, 758-761.

Brown, M., Korte, M., Holme, R., Wardinski, I., Gunnarson, S., 2018. Earth's magnetic field is (probably) not reversing. Proc. Natl. Acad. Sci. 115(20), 5111-5116.

Campuzano, S. A., Gómez-Paccard, M., Pavón-Carrasco, F., Osete, M. L., 2019. Emergence and evolution of the South Atlantic Anomaly revealed by the new paleomagnetic reconstruction SHAWQ2k. Earth Planet. Sci. Lett. 512, 17-26.

Casadio, S., Arino, O., 2011. Monitoring the South Atlantic Anomaly using ATSR instrument series. Adv. Space Res. 48(6), 1056-1066.

Christensen, U., Aubert, J., Hulot, G., 2010. Conditions for Earth-like geodynamo models. Earth Planet. Sci. Lett. 296, 487-496. 
Constable, C., 2007. Centennial- to millennial-scale geomagnetic field variations. In: Kono, M. (Ed.), Treatise on Geophysics. Vol. 5. Elsevier Science.

Davies, C., Constable, C., 2014. Insights from geodynamo simulations into long-term geomagnetic field behaviour. Earth planet. Sci. Lett. 404, 238-249.

De Santis, A., Qamili, E., Wu, L., 2013. Toward a possible next geomagnetic transition? Nat. Hazards Earth Syst. Sci. 13, 3395-3403.

Finlay, C., 2008. Historical variation of the geomagnetic axial dipole. Phys. Earth Planet. Inter. $170,1-14$.

Finlay, C. C., Maus, S., Beggan, C. D., Bondar, T. N., Chambodut, A., Chernova, T. A., Chulliat, A., Golovkov, V. P., Hamilton, B., Hamoudi, M., Holme, R., Hulot, G., Kuang, W., Langlais, B., Lesur, V., Lowes, F. J., Lühr, H., Macmillan, S., Mandea, M., McLean, S., Manoj, C., Menvielle, M., Michaelis, I., Olsen, N., Rauberg, J., Rother, M., Sabaka, T. J., Tangborn, A., Tøffner-Clausen, L., Thébault, E., Thomson, A. W. P., Wardinski, I., Wei, Z., Zvereva, T. I., 2010. International Geomagnetic Reference Field: the eleventh generation. Geophys. J. Int. 183, 1216-1230.

Gastine, T., Aubert, J., Fournier, A., 2019. Dynamo-based limit to the extent of a stable layer atop Earth's core. Geophys. J. Int. 200, 1-16.

Gillet, N., Barrois, O., Finlay, C. C., 2015. Stochastic forecasting of the geomagnetic field from the COV-OBS.x1 geomagnetic field model, and candidate models for IGRF-12. Earth Plan. Space 67:71, doi: 10.1186/s40623-015-0225-z.

Gubbins, D., 1987. Mechanism for geomagnetic polarity reversals. Nature 326, 167-169.

Gubbins, D., Willis, P., Sreenivasan, B., 2007. Correlation of Earth's magnetic field with lower mantle thermal and seismic structure. Phys. Earth Planet. Inter. 162, 256-260.

Hare, V., Tarduno, J., Huffman, T., Watkeys, M., Thebe, P., Manyanga, M., Bono, R., Cottrell, R., 2018. New archeomagnetic directional records from Iron Age southern Africa (ca. 425-1550 CE) and implications for the South Atlantic Anomaly. Geophys. Res. Lett. 45, 1361-1369. 
Hartmann, G., Pacca, I., 2009. Time evolution of the South Atlantic Magnetic Anomaly. An. Acad. Bras. Cien. 81, 243-255.

Hartmann, G. A., Poletti, W., Trindade, R. I. F., Ferreira, L. M., 2019. New archeointensity data from South Brazil and the influence of the South Atlantic Anomaly in South America. Earth Planet. Sci. Lett. 512, 124-133.

Hellio, G., Gillet, N., 2018. Time-correlation-based regression of the geomagnetic field from archeological and sediment records. Geophys. J. Int. 214(3), 1585-1607.

Huguet, L., Amit, H., Alboussière, T., 2018. Geomagnetic dipole changes and upwelling/downwelling at the top of the Earth's core. Front. Earth Sci. 6:170, doi:10.3389/feart.2018.00170.

Jackson, A., Jonkers, A., Walker, M., 2000. Four centuries of geomagnetic secular variation from historical records. Phil. Trans. R. Soc. Lond. A358, 957-990.

Olson, P., Amit, H., 2006. Changes in earth's dipole. Naturwissenschaften 93, 519-542.

Pavón-Carrasco, F., De Santis, A., 2016. The South Atlantic Anomaly: The key for a possible geomagnetic reversal. Front. Earth Sci. 4:40, doi: 10.3389/feart.2016.00040.

Schaefer, R. K., Paxton, L. J., Selby, C., Ogorzalek, B., Romeo, G., Wolven, B., Hsieh, S., 2016. Observation and modeling of the South Atlantic Anomaly in low Earth orbit using photometric instrument data. Space Weather 14, 330-342.

Tarduno, J., Watkeys, M., Huffman, T., Cottrell, D., Blackman, E., Wendt, A., Scribner, A., Wagner, C., 2015. Antiquity of the South Atantic Anomaly and evidence for top-down control on the geodynamo. Nat. Commun. 6, 7865, doi:10.1038/ncomms8865.

Terra-Nova, F., Amit, H., Choblet, G., 2019. Preferred locations of weak surface field in numerical dynamos with heterogeneous core-mantle boundary heat flux: consequences for the South Atlantic Anomaly. Geophys. J. Int. 217, 1179-1199. 
357 Terra-Nova, F., Amit, H., Hartmann, G. A., Trindade, R. I. F., Pinheiro, K. J., 2017. Relating 358 the South Atlantic Anomaly and geomagnetic flux patches. Phys. Earth Planet. Inter. 266, $359 \quad 39-53$.

${ }_{360}$ Trindade, R., Jaqueto, P., Terra-Nova, F., Brandt, D., Hartmann, G. A., Feinberg, J., Strauss, 361 B. E., Novello, V. F., Cruz, F. W., Karmann, I., Cheng, H., Edwards, R. L., 2018. Speleothem 362 record of geomagnetic South Atlantic Anomaly recurrence. Proc. Nat. Acad. Sci. 115, $363 \quad 13198-13203$.

364 Zossi, B., Fagre, M., Amit, H., Elias, A. G., 2020. Geomagnetic field model indicates shrinking 365 northern auroral oval. J. Geophys. Res., accepted. 


\section{Figures}

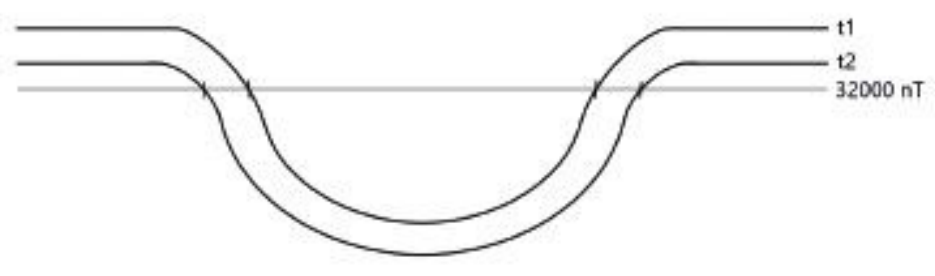

\section{Figure 1}

Schematic illustration of a cross-section of the surface field intensity under a global decrease in field intensity from time 11 to time $t 2$. In this scenario, the apparent SAA area based on S0 (1) increases.

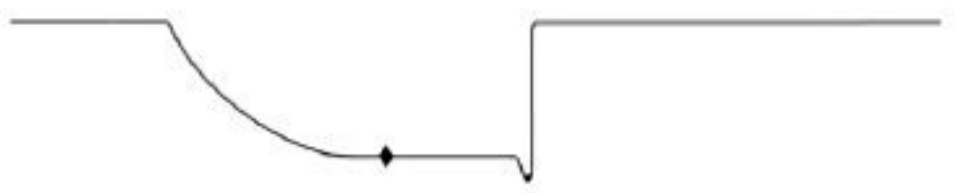

\section{Figure 2}

Schematic illustration of a cross-section of the surface field intensity with an anisotropic configuration. The SAA center based on the minimum (Min, denoted by a point) does not represent well the center of the anomaly. The center of mass method $\mathrm{CM} 2$ gives an adequate center denoted by a diamond. 

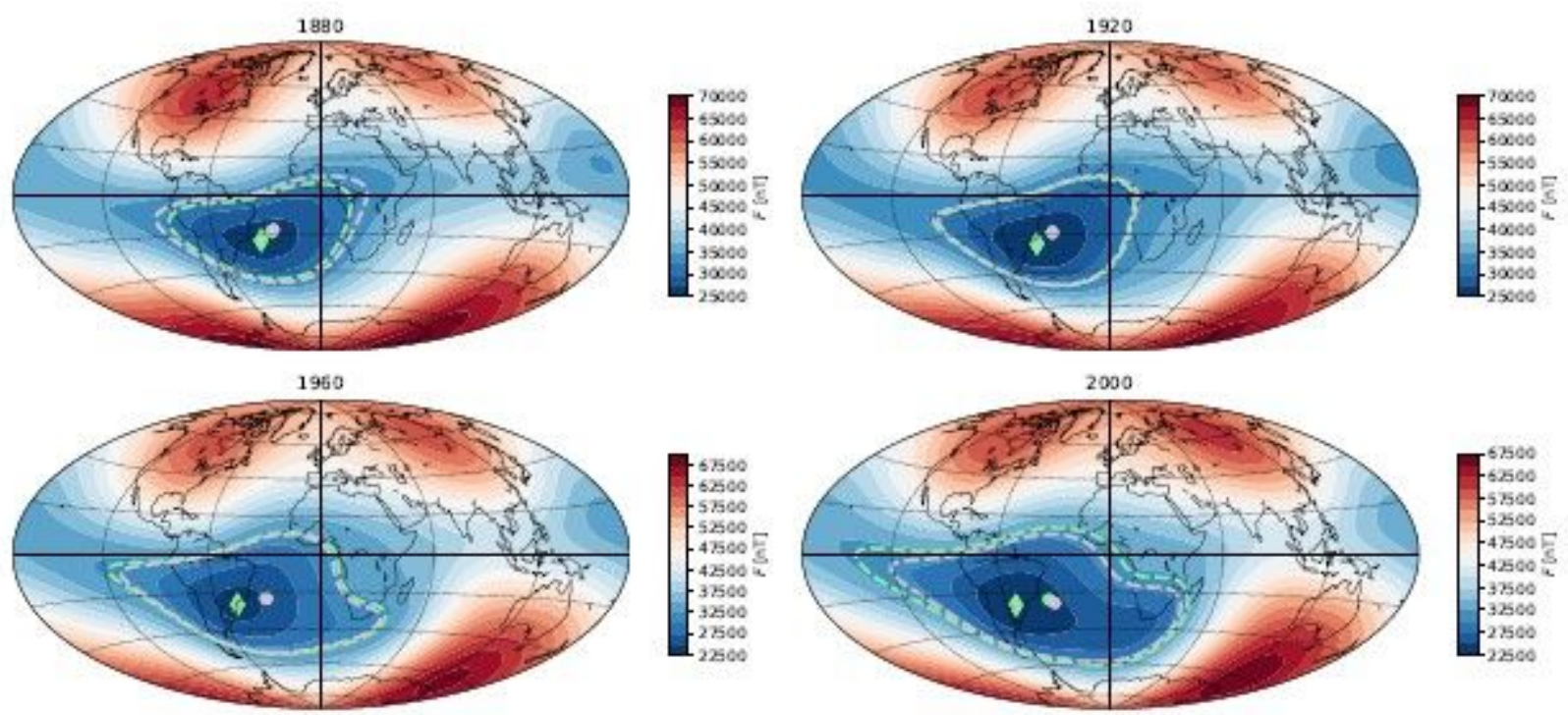

\section{Figure 3}

Geomagnetic field intensity at Earth's surface for the mean model of COV-OBS.x1 at four snapshots (Gillet et al., 2015). Dashed green contours denote the S0 area, dashed purple contours denote the S1 area. Green diamonds denote the Min center, green and purple diamonds denote the centers of mass CMO and $\mathrm{CM} 1$, respectively. Note the different scales from top to bottom.

(a)

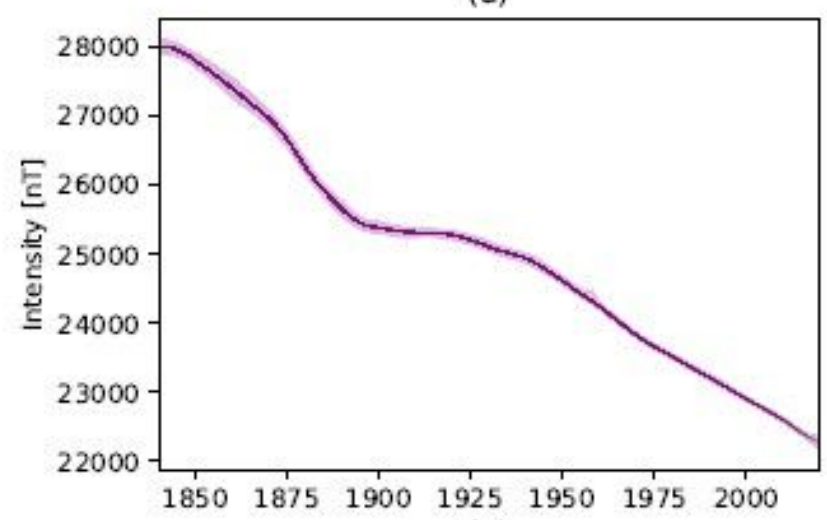

(c)

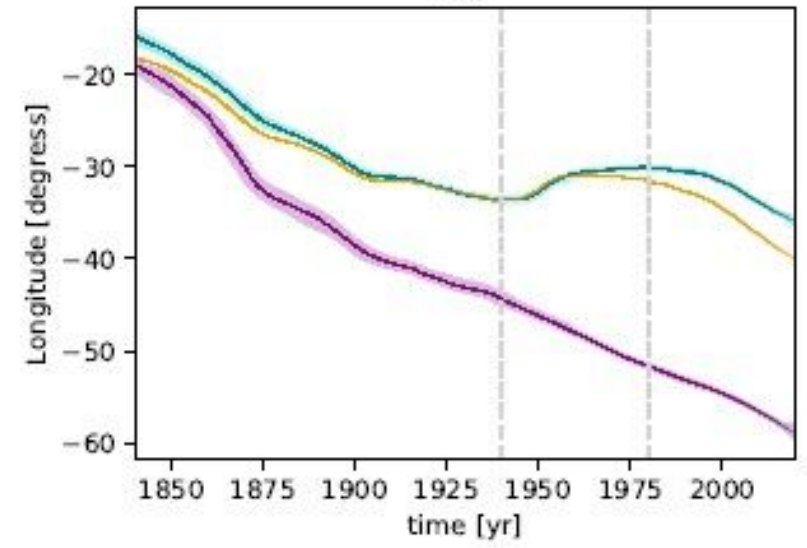

(b)

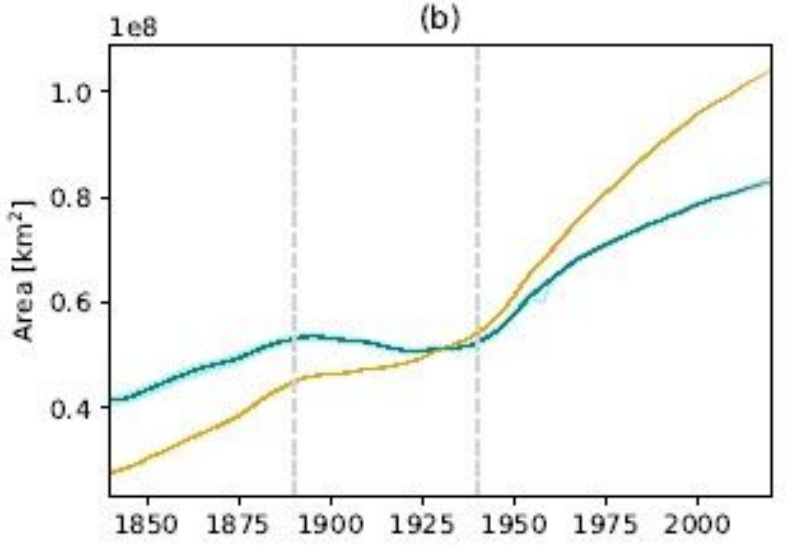

(d)

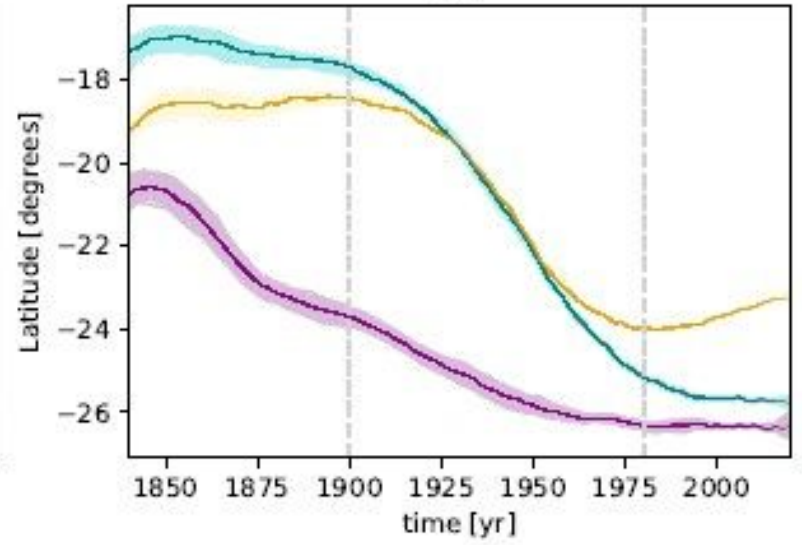




\section{Figure 4}

SAA characterization vs. time for the period 1840-2020. Dark lines denote the mean model of COVOBS.x1, light envelopes denote the 100 realizations of the ensemble. (a) Minimum intensity; (b) Area based on S0 (yellow) and S1 (green); (c) Longitude of center based onMin (purple), CM0 (yellow) and CM1 (green); (d) Latitude of center based onMin (purple), CM0 (yellow) and CM1 (green). Vertical dashed lines highlight special events of SAA area decrease (b), SAA center eastward drift (c) and SAA center rapid southward drift (d).
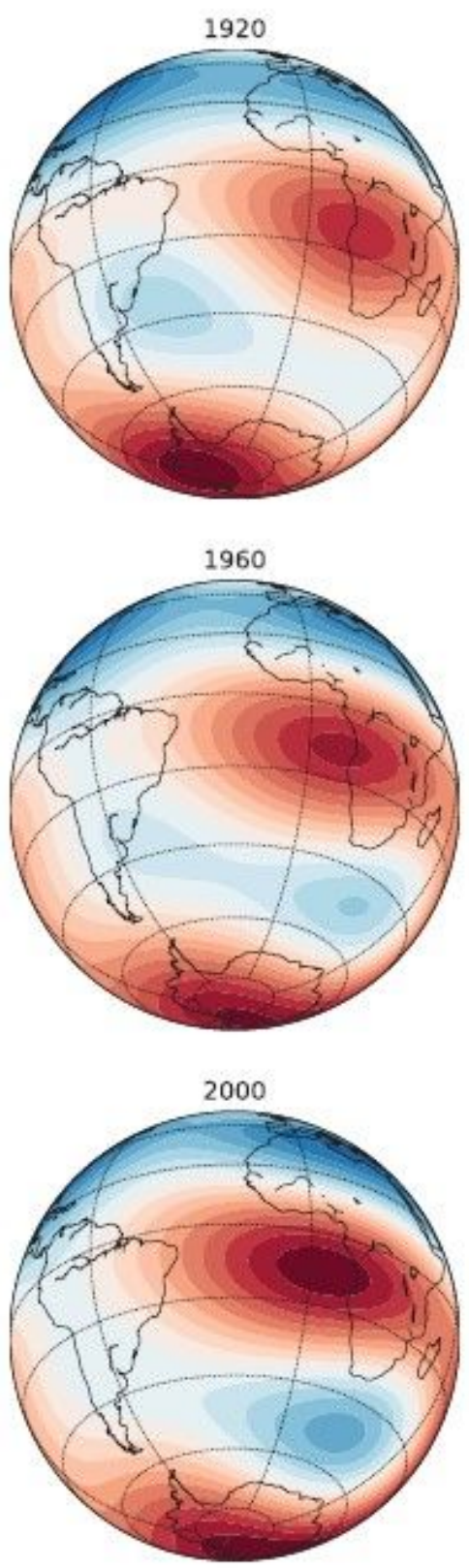
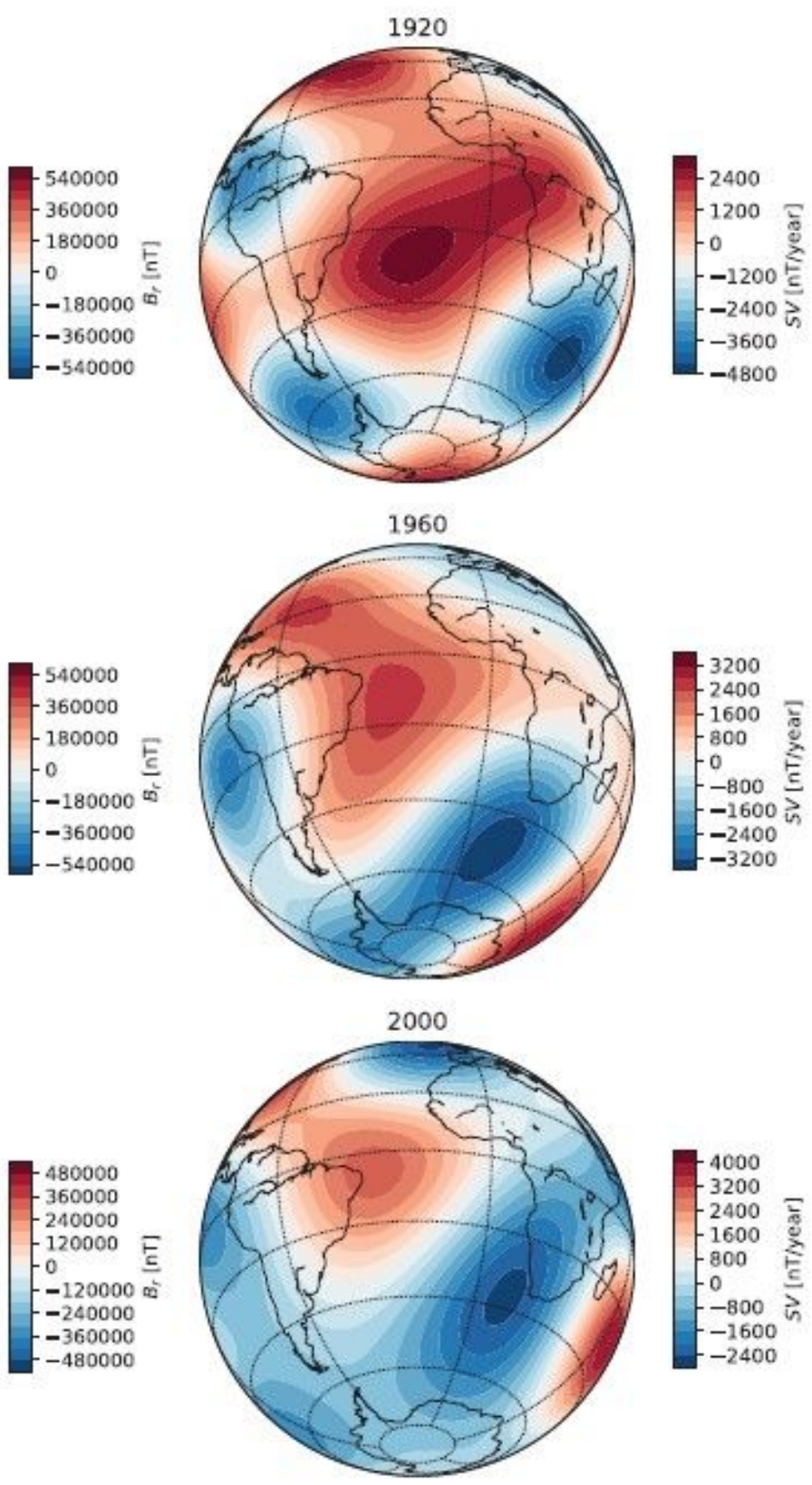
Radial geomagnetic field (left) and its secular variation (right) at the core-mantle boundary for the mean model of COV-OBS.x1 in 1920 (top), 1960 (middle) and 2000 (bottom). All models are expanded until spherical harmonic degree and order 5 . All maps are centered at $20^{\circ} \mathrm{W} 30^{\circ} \mathrm{S}$, i.e. on the South Atlantic. Note the different scales.
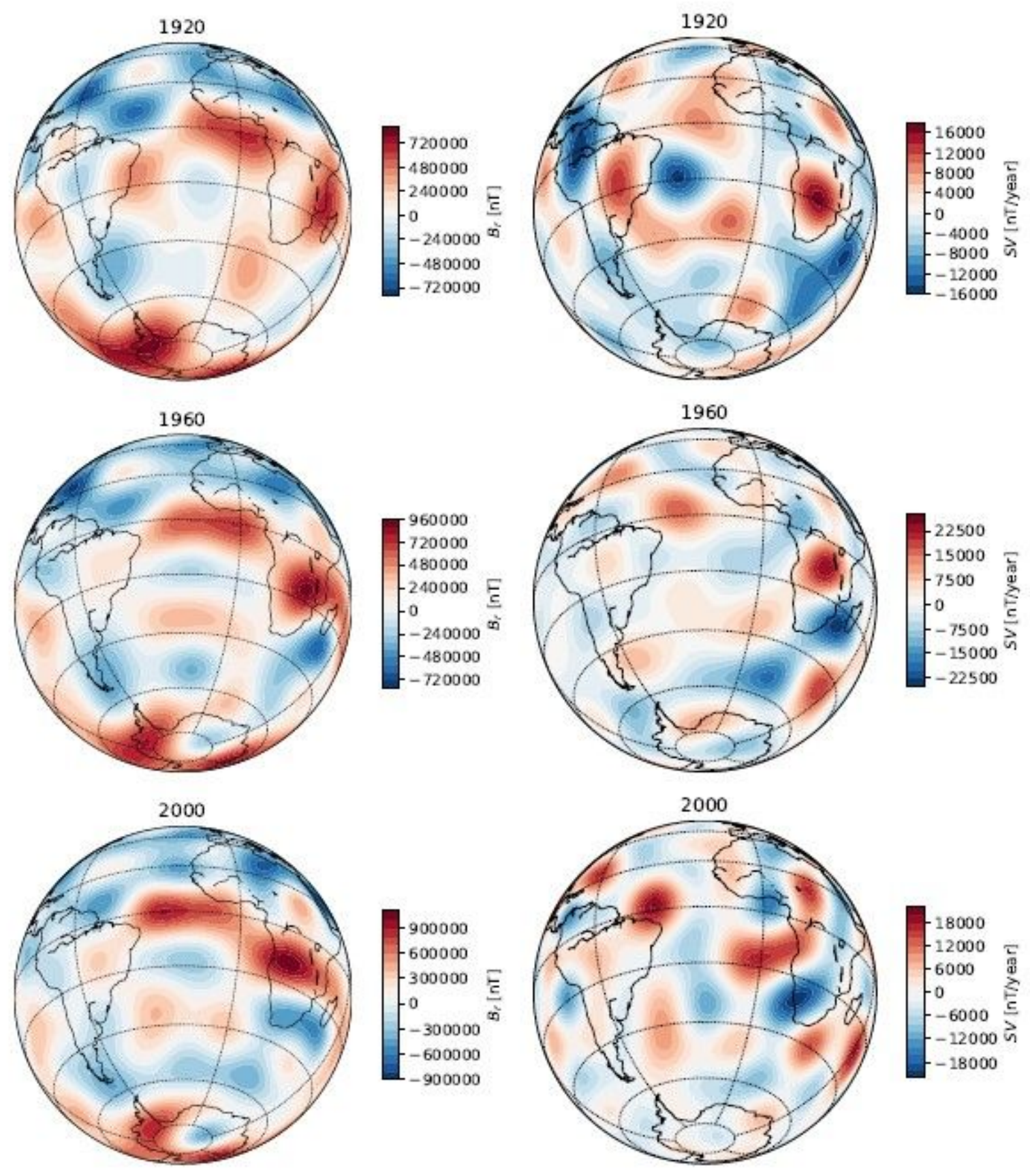

\section{Figure 6}

As in Fig. 5 but expanded until spherical harmonic degree and order 14. 


\section{Supplementary Files}

This is a list of supplementary files associated with this preprint. Click to download.

- F2000.jpg 